\title{
GLAD!
}

Revue sur le langage, le genre, les sexualités

\section{Débat sur le travail et le soin : de quoi parlons-nous lorsque nous parlons de travail de soin?}

Debatiendo sobre el trabajo y los cuidados: ¿De qué hablamos cuando nos

referimos al trabajo de cuidados?

Andrea Barbara Blazsek, Maria Celeste Linardelli, Daniel Garcia et Maria Agustina Diez

Traducteur : Atelier de traduction FELiCiTE

\section{OpenEdition}

Journals

Édition électronique

URL : http://journals.openedition.org/glad/2077

DOI : $10.4000 /$ glad.2077

ISSN : 2551-0819

Éditeur

Association GSL

Référence électronique

Andrea Barbara Blazsek, Maria Celeste Linardelli, Daniel Garcia et Maria Agustina Diez, « Débat sur le travail et le soin : de quoi parlons-nous lorsque nous parlons de travail de soin ? », GLAD! [En ligne], 09 | 2020, mis en ligne le 20 décembre 2020, consulté le 20 janvier 2021. URL : http:// journals.openedition.org/glad/2077 ; DOI : https://doi.org/10.4000/glad.2077

Ce document a été généré automatiquement le 20 janvier 2021.

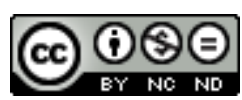

La revue GLAD! est mise à disposition selon les termes de la Licence Creative Commons Attribution Pas d'Utilisation Commerciale - Pas de Modification 4.0 International. 


\section{Débat sur le travail et le soin : de quoi parlons-nous lorsque nous parlons de travail de soin?}

Debatiendo sobre el trabajo y los cuidados: ¿De qué hablamos cuando nos referimos al trabajo de cuidados?

Andrea Barbara Blazsek, Maria Celeste Linardelli, Daniel Garcia et Maria Agustina Diez

Traduction : Atelier de traduction FELiCiTE

Traducteur-es : Atelier de traduction FELiCiTE

(rattaché au laboratoire Triangle - UMR 5206)

composé de : Blanche Turck, Charlie Brousseau,

Marie Bilau, Estelle Fisson, Emilie Fernandez,

Andrea Rico, Marianna Cesano, Cécile Pilgram,

Bethsabée Maire

Relecture et coordination : Charlie Brousseau

Cette communication est issue des Journées nationales de recherche en sciences sociales de la Universidad Nacional de Cuyo - 25 et 26 aout 2016, « Perspectives actuelles de recherche en sciences sociales : problématiques, perspectives épistémologiques et orientations théorico-méthodologiques ".

La présente communication s'est élaborée dans le champ des projets de recherche suivants : «Travail productif et reproductif en tension : trajectoires professionnelles de femmes avec des enfants sur le marché du travail de Mendoza » (Projet soutenu et financé par la Universidad Nacional de Cuyo, Secretaría de Ciencia, Técnica y Posgrado, période 2013-2015, directrice : Andrea B. Blazsek, co-directrice : Azucena Reyes Suárez) et « Travail, genre et classe : trajectoires professionnelles et stratégies de soin des femmes avec des enfants, membres de différents classes sociales à Mendoza » (Projet soutenu et financé par 1 Universidad Nacional de Cuyo, Secretaría de Ciencia, Técnica y Posgrado, période 
2016-2018, directrice : Andrea Barbara Blazsek, co-directrice : Maria Agustina

Diez)

Autres membres de l'équipe de recherche : Flavia Carina Espinosa, María

Valentina Saenz, Eugenia Peiretti, María Laura San Martín, Natalia Alonso

1 Tout concept, puisqu'il est une construction, revêt différentes significations en fonction des points de vue théoriques depuis lesquels on le définit ainsi que des caractéristiques socio-historiques du contexte auquel il se rapporte. Les concepts, de même que les phénomènes qu'ils cherchent à représenter, ne sont ni immuables ni inaltérables. Ne pas reconnaitre la variabilité que les différents phénomènes ont subie au fil du temps, même si on continue à les désigner avec les mêmes termes, c'est leur donner un rôle essentialiste et naturalisé. Des notions comme le travail, la domesticité, le travail domestique, la maternité, le soin ${ }^{1}$, ont des significations variables selon les périodes historiques et les perspectives théoriques dans lesquelles elles se cristallisent.

2 Le sens commun, tout comme certains discours scientifiques, tendent à naturaliser l'usage de concepts déterminés, dissimulant ainsi les processus socio-historiques qui ont conduit à leur genèse et leur développement. Les sciences sociales sont perméables à ce qu'on appelle la « sociologie spontanée » et à l'usage acritique de prénotions qui se cachent sous l'apparence d'une élaboration scientifique. C'est en ce sens que Pierre Bourdieu remarque: «Le langage ordinaire, qui, parce qu'ordinaire, passe inaperçu, enferme, dans son vocabulaire et sa syntaxe, toute une philosophie pétrifiée du social toujours prête à resurgir des mots communs ou des expressions complexes construites avec des mots communs que le sociologue utilise inévitablement » (1983 : 36-37).

3 En accord avec cette mise en garde, nous affirmons la nécessité de reconnaitre le caractère socio-historique des différentes définitions autour de ce qu'est le travail et de quelles activités celui-ci recouvre.

4 Nous rejoignons Enrique de la Garza Toleda (2001) sur l'idée que les limites qui ont tendance à s'établir entre le travail et le non-travail ne sont pas naturelles, elles se définissent socialement et sont sujettes à des modifications lorsque les relations entre les forces économiques et sociales changent. Pour différencier ce qui relève du travail et du non-travail, il ne suffit pas de considérer le type d'activité ou l'objet du travail : il est nécessaire de prendre en compte ses articulations avec certaines relations sociales de subordination, de coopération, d'exploitation ou d'autonomie. La notion de travail n'est pas anhistorique, il s'agit d'une construction sociale façonnée par les relations de pouvoir et de domination dans une configuration sociale déterminée.

\section{Quelques réflexions sur les notions de travail productif et reproductif}

5 Marx (Le Capital, 1867) soutient que dans le capitalisme n'est productif que l'ouvrier qui travaille pour rentabiliser le capital. Dans cette affirmation, le concept de travail productif se situe dans une relation sociale de production déterminée, historiquement configurée. En effet, dans le mode de production capitaliste, la notion de travail s'est circonscrite aux activités qui produisent les biens et les services avec une valeur d'échange dans le marché, c'est-à-dire celles qui contribuent directement à l'accumulation du capital. 
6 Les approches économiques néoclassiques de la fin du $\mathrm{XIX}^{\mathrm{e}}$ siècle et du $\mathrm{XX}^{\mathrm{e}}$ siècle, qui perdurent jusqu'à aujourd'hui et qui ont imprégné le sens commun, adoptent le concept restreint de travail comme se limitant à l'activité économique rémunérée dans le domaine du marché (Benería 1999). C'est ainsi qu'on a désigné par le terme de «travail » uniquement la forme dominante du travail dans le système capitaliste, c'està-dire le travail salarié qui produit directement pour le capital (Himmelweit 2005).

7 Les approches économiques orthodoxes ont établi une division stricte entre travail/ non-travail, en se basant exclusivement sur la perception ou non d'une rémunération résultant de la vente de la force de travail du marché. Les formes de travail qui s'éloignent de la définition dominante ont été marginalisées par rapport à l'héritage conceptuel orthodoxe des sciences sociales, ce qui a rendu compliqué de les mesurer empiriquement.

8 Ainsi, différentes formes de travail qui ne correspondent pas au canon du travail salarié rémunéré ont rejoint la catégorie de non-travail, et les personnes assignées à ces activités (par exemple les femmes au foyer) ont été désignées comme une population économiquement inactive. Par conséquent, dans les statistiques officielles, ces formes de travail sont devenues insignifiantes d'un point de vue économique et les personnes qui se consacrent à ces activités ont progressivement constitué la catégorie des inactif/ves.

9 Il a fallu et il faut toujours de longs débats pour que des modalités de travail comme celles de la subsistance, du domestique, du bénévolat, de l'autoconsommation, soient considérées comme du travail pour commencer à mieux les mesurer empiriquement et mieux les valoriser économiquement. L'emploi ou le travail marchand rémunéré est devenu le travail et l'utilisation restrictive de ce concept s'est naturalisée, tant dans les traditions théoriques des approches dominantes des sciences sociales, que dans les indicateurs statistiques officiels qui répondent à la définition standard de la comptabilité nationale.

10 Les organismes internationaux ont été chargés de diffuser des définitions conceptuelles et opérationnelles du travail, réduit à l'activité marchande rémunérée, à travers des recommandations effectuées pour l'Organisation Internationale du Travail et les Nations Unies (entre autres), à partir des années 1950. L'OIT réduit le fait statistique de travailler au groupe des personnes ayant un emploi, c'est-à-dire, des personnes qui réalisent un travail pour un salaire ou pour en retirer un bénéfice. Plus avant, on définit la population économiquement active comme " toutes les personnes d'un sexe ou de l'autre qui contribuent par leur travail à produire des biens et des services économiques", en essayant d'établir, en plus de la force de travail effectivement employée, le nombre de personnes au chômage et la main-d'œuvre disponible pour travailler (OIT, cité dans Benería 1999: 322), s'agissant toujours du travail circonscrit à la sphère marchande dans le contexte socio-historique spécifique aux économies des pays occidentaux durant la période postérieure à la Seconde Guerre mondiale.

11 Dès lors, ce qui est conceptualisé communément comme travail par le champ académique orthodoxe, par les institutions gouvernementales et par le sens commun, a pour origine le processus de consolidation du capitalisme et il faut donc un effort de dénaturalisation et de situation dans un processus socio-historique spécifique. 
12 Les études historiques de la vie quotidienne ont montré que pendant l'époque préindustrielle, le foyer se constituait d'une unité productive autant que reproductive et que la division sexuelle du travail prenait des modalités variées selon les différents contextes socio-économiques (Carrasco, Borderías et Torns 2011). Pendant le processus d'industrialisation capitaliste, l'unité productive se sépare de l'unité domestique, tandis que le processus de salarisation du travail, mis au service du capital, commence.

13 Seccombe (1974) parle de la scission du processus général du travail, dans la mesure où le foyer se vide de ses fonctions productives et se circonscrit aux processus de consommation à l'intérieur du foyer et à la reproduction de la vie, alors que la production de marchandises se déplace dans les ateliers et les usines. C'est au cours des $\mathrm{XVII}^{\mathrm{e}}$ et $\mathrm{XVIII}^{\mathrm{e}}$ siècles qu'on peut situer temporellement l'apparition de la notion de travail, en tant que celui-ci est considéré comme une activité extra domestique, industrielle, salariée, marchande et rémunérée, en excluant de ce concept toutes les dimensions du travail qui ont à voir avec les activités domestiques, de subsistance et d'autoproduction.

14 À mesure que le capitalisme se renforce, l'unité domestique se vide de la fonction productive. Cela se fait en dépit du fait que, à partir de 1945 seulement, avec la montée des États-providence dans les pays occidentaux et le dénommé "Âge d'Or du capitalisme ", on constate dans les pays occidentaux une élimination quasi totale des activités productives à l'intérieur des foyers. Jusqu'en 1945, comme le souligne Mies (1981), tant dans les foyers ruraux qu'urbains des pays du Nord occidental, on continuait à produire des biens pour l'autoconsommation (la culture des légumes, l'élevage des animaux, la fabrication du pain, etc.). Après la Seconde Guerre mondiale, pour la première fois, l'augmentation des salaires des employé-es et l'expansion de la consommation de masse conduisent à une marchandisation des différents biens et services produits à l'intérieur des foyers.

15 Les activités d'entretien et de reproduction de la vie, réalisées dans l'unité domestique/le foyer, sont devenues du non-travail, de telle sorte que le travail réalisé dans les foyers et celui effectué sur le marché commencent à prendre des chemins opposés, invisibilisant et dévalorisant le premier face à un travail marchand rémunéré.

16 La division du processus général du travail entre le foyer et l'usine produit aussi une forme spécifique de division sexuée du travail entre les femmes et les hommes. De cette manière, une grande partie du non-travail dans le capitalisme (reproductif dans les foyers, de subsistance, etc.) est réalisé par les femmes, tandis que le travail salarié s'est masculinisé, en particulier vers la fin du XIx ${ }^{e}$ siècle et la première moitié du Xx $x^{e}$ siècle.

17 À cet égard, différentes auteures féministes affirment que le capitalisme a renforcé les structures patriarcales, en confinant les femmes à la sphère privée (au foyer et au soin des membres de la famille). La dichotomie public/privé a mené à l'association des hommes au pouvoir, à l'autorité, au public et au travail et l'association des femmes à la soumission, à la dépendance, au familier, au privé et au domestique.

$18 \mathrm{Au} \mathrm{xx}^{\mathrm{e}}$ siècle, le travail se référant à l'emploi et les droits qui lui sont associés constituaient déjà l'élément central des États-providence de l'après-guerre. Ainsi, une citoyenneté salariée et industrielle (masculine) se consolide, porteuse de droits sociaux et du travail, pendant que les femmes au foyer restent exclues de ces droits, processus qui renforce et alimente les inégalités hommes/femmes. Effectivement, les critiques de certaines auteures féministes, comme Jane Lewis en Angleterre, Nancy Folbre aux 
États-Unis et Diane Sainsbury dans les pays scandinaves, contre les régimes des Étatsprovidence, montrent que l'amplification du droit du travail et du droit social n'est pas parvenue à éliminer les inégalités entre les hommes et les femmes, mais au contraire, les maintient ou les renforce : tel est le cas des brèches salariales qui n'ont pas disparu, même dans les pays nord-européens.

19 La conceptualisation restreinte du travail a participé à consolider le travail rémunéré comme objet d'étude des différentes disciplines des sciences sociales, et à circonscrire le dénommé monde du travail aux phénomènes qui se produisent autour de l'activité professionnelle marchande et rémunérée.

20 L'incorporation d'une définition plus large, qui permet de retrouver les autres formes de travail qui ne sont pas développées dans le domaine marchand et la problématisation du travail reproductif en tant qu'objet d'étude des sciences sociales, s'est faite lentement. En effet, l'invisibilisation du travail reproductif dans le capitalisme affecte également les possibilités de son appréhension théoricoconceptuelle.

21 Marx, bien que traitant dans ses œuvres du procédé de reproduction de la force de travail, ne développe pas explicitement la question du travail domestique et reproductif dans sa contribution à l'économie capitaliste.

22 Carrasco, Borderías et Torns (2011) soutiennent que les penseurs de l'économie classique n'ont pas étudié le travail reproductif en tant que catégorie économique, mais ils ont reconnu son importance dans les procédés de reproduction de la force de travail.

23 En revanche, les approches économiques marginalistes et néoclassiques de la fin du $\mathrm{XIX}^{\mathrm{e}}$ siècle ont occulté la contribution économique et ont installé jusqu'à aujourd'hui la définition $\mathrm{du}$ travail qui prévaut dans différents domaines académiques, gouvernementaux et reproductifs pour le sens commun : les activités qui se réalisent dans le domaine marchand et qui reçoivent une certaine rémunération. Même si l'économie néoclassique n'a pas tenté de produire beaucoup de connaissances théoricoempiriques autour du travail reproductif, elle n'a pas complètement ignoré l'apport de celui-ci à la reproduction sociale, bien qu'elle le circonscrive aux activités "désintéressées", altruistes, tel qu'on peut le constater dans l'affirmation suivante énoncée par le fondateur économique néoclassique, Alfred Marshall, dans ses Principes d'économie politique (1890) : «Le capital le plus précieux, c'est celui que représentent les êtres humains : et la partie la plus précieuse de ce capital provient des soins et de l'influence de la mère, du moins tant qu'elle conserve ses instincts de tendresse et de désintéressement » (Marshall, cité dans Federici 2013:71).

24 Déjà dans les années 1960, et de façon plus importante encore dans les années 1970, ces conceptualisations référant au travail se voient questionnées de façon importante: d'une part, de la part des enquêteurs/trices dédiées à l'étude les réalités économiques du dit tiers-monde ou des pays périphériques, mettant l'emphase sur l'importance des activités de subsistance pour les économies de ces pays, et, d'autre part, de la part des chercheurs/ses tant féministes que marxistes qui commencent à révéler les caractéristiques du travail domestique dans l'ordre social capitaliste, particulièrement son caractère féminin, son occultation en tant que travail et sa configuration comme instrument de domination des hommes sur les femmes.

25 Ces inquiétudes tant théoriques que politiques donnent lieu au dénommé « débat sur le travail domestique » qui implique différent-es chercheur/ses d'Europe et d'Amérique 
(Silvia Federici, Mariarosa Dalla Costa, Juliet Mitchell, Jean Gardiner, Maxine Molineux, Christine Delphy, John Harrison, Mary Goldsmith, Victoria Benholdt-Thomsen, Margaret Coulson, Susan Himmelweit, Lourdes Benería, Teresita de Barbieri, Isabel Larguía, John Dumoulin, Wally Seccombe, Margaret Benston, Heidi Hartman, Shulamith Firestone). Il s'agit de «théoriser et politiser le travail domestique » comme le dit Silvia Federici dans son livre Point zéro: propagation de la révolution dans lequel la féministe italienne réunit plus de trente ans de réflexions et d'enquêtes sur la nature du travail domestique, la reproduction sociale et les luttes des femmes dans ce domaine.

26 Ce débat se déploie autour de différents aspects du travail domestique, tels que la définition, le contenu et la forme de ce travail, sa relation avec le mode de production capitaliste et de façon plus générale son rôle au sein de l'ordre social capitaliste. Outre ces préoccupations de nature académique, le débat lui-même renferme un caractère politique, permettant une reconnaissance et une mise en valeur du travail domestique, tout en favorisant la lutte pour l'émancipation des femmes, l'élimination des différentes formes d'oppression dont les femmes souffrent et des inégalités de genre (Rodríguez et Cooper, 2005).

27 Karina Batthyany signale que les remises en question de la notion traditionnelle de travail, considérée exclusivement dans le champ de la production et de l'échange marchand de biens et de services, mènent à une rupture théorico-méthodologique avec la manière classique d'aborder la sociologie du travail. Cette rupture permet de redéfinir et d'étendre la notion de travail, afin de mettre en lumière le caractère socialement construit tant des relations de genre que de la division sexuelle du travail.

28 Si le travail domestique renvoie bien à une série d'activités quotidiennes qui ont lieu dans les foyers/familles avec pour but la production et la reproduction de la vie humaine, il s'agit d'après Teresa Torns (2008: 57) d'un concept flou et controversé quant à sa dénomination (travail du foyer, reproductif, de soin), son contenu et sa valeur. Sur ce dernier point, les débats ont été intenses et n'ont pas permis de déterminer si le travail domestique produit seulement des valeurs d'usage ou s'il contribue aussi à la valeur de la force de travail, produisant une marchandise.

29 Teresita de Barbieri (1978: 110) énumère un ensemble de tâches, "qu'on ne nomme plus », qui se retrouvent dans le travail domestique : cuisiner, laver le linge, repasser, nettoyer la maison, s'occuper des enfants, les nourrir, les coucher, les conduire d'un endroit à l'autre, et la liste pourrait continuer. L'objectif principal de ce travail consiste à s'occuper de la consommation individuelle de chaque membre du foyer/de la famille qui requiert, en grande partie, la transformation des marchandises acquises grâce au salaire sur le marché, pour être consommées au sein du foyer.

30 Barbieri affirme que «le travail domestique permet que le travailleur maintienne sa capacité de vendre sa force de travail et qu'il y ait quelqu'un pour le remplacer quand il meurt ou part à la retraite» (Barbieri 1978 : 111).

31 En résumé, le travail domestique assure le maintien, la reconstitution et la reproduction de la force de travail actuelle et future.

32 Isabel Larguia et John Dumoulin, pionnier-es dans les études de la reproduction de la force de travail, classent le contenu du travail domestique en deux catégories: d'un côté, la reproduction générationnelle de la force de travail qui inclut la reproduction strictement biologique, l'éducation et la socialisation de la force de travail et, de l'autre côté, le maintien quotidien de la force de travail qui consiste à transformer les moyens 
de subsistance (revenus) en valeurs d'usage pour la consommation et à offrir un soutien émotionnel pour atténuer la déshumanisation dans le lieu de travail.

En regroupant les tâches nécessaires à la reproduction de la force de travail, la notion de travail domestique est remplacée par la suite par le concept de travail reproductif ou de la reproduction, qui étend la définition du travail domestique limité exclusivement au domaine du foyer à un contexte extradomestique et extrafamilial.

34 Le travail reproductif s'inscrit dans le processus plus général de la reproduction sociale qui est lié à la constitution et re-création du social (sa permanence et ses évolutions) dans différents domaines: individuel, familial, collectif et sociétal. Il s'agit d'un processus complexe qui prend en compte des éléments biologiques autant que des éléments sociaux. Ces derniers renvoient aux aspects matériels et symboliques qui sont liés à l'économie, à la démographie, et à la politique (Oliveira et Salles 1987).

35 Carrasquer, Torns et al. (1998) définissent le travail de la reproduction comme l'ensemble des activités qui permettent la reproduction biologique, sociale et idéologique de la force de travail et proposent les dimensions suivantes: le travail d'approvisionnement; le travail de structuration du foyer; le souci des charges reproductives passées, présentes et futures; l'organisation du fonctionnement du foyer ; le travail de médiation entre le foyer et l'ensemble des services offerts par l'État et les institutions publiques, le travail de médiation dans les relations affectives; le travail de maintien de l'image du couple. Concernant le caractère historique du travail de reproduction, les auteur-es préviennent que ces dimensions peuvent varier selon le contexte socio-historique d'analyse.

\section{Brève trajectoire de la conceptualisation du soin et du travail de soin dans l'espace européen}

36 Alors que le débat sur le travail domestique et reproductif se développe à l'intersection des perspectives marxistes et féministes, le concept de soin commence à prendre forme dans les années 1980 en Europe, en sciences sociales. Ce concept s'incarne dans trois courants de pensée différents, respectivement dans les pays scandinaves, en Italie et en Angleterre, bien que ceux-ci s'influencent mutuellement.

37 La première de ces origines peut être placée dans les pays scandinaves, dans le cadre des études sur l'État-providence et les caractéristiques de ce qu'on appelle modèle nordique de l'État-providence (Alestalo et al. 2009). Différentes recherches menées par des politologues féministes scandinaves (Helga Hernes, Diane Sainsbury, etc.) à partir des années 1970 mettent en évidence que, si ces sociétés ont réussi à réduire significativement les inégalités économiques, éducatives et sociales par rapport aux autres pays capitalistes occidentaux, ce n'est pas le cas pour ce qui concerne les inégalités de genre, car les politiques mises en œuvre par l'État-providence ont tendance, généralement, à être neutres du point de vue du genre.

38 Attentives à cette situation, les féministes scandinaves soulignent la contribution indispensable des femmes au bien-être social à travers le soin de la vie, notamment dans les pays nordiques où la reproduction sociale, habituellement cantonnée à la sphère privée, dépasse les frontières du privé et s'introduit dans la sphère publique, où elle est amplement mise en commun (Leira et Saraceno 2006). En même temps, dans un contexte de délégitimation croissante de l'État-providence par la pensée néolibérale qui 
se consolide dans les années 1980, des voix s'élèvent pour mettre en cause la socialisation et la professionnalisation des services de soin et plaident en faveur de leur re-privatisation, c'est-à-dire leur réinsertion dans le milieu privé/familial ou communautaire (Waerness 1996). C'est dans le cadre de ces discussions qu'un travail pionnier naît sur le soin (concept dénommé omsorg dans les langues scandinaves), rédigé en 1984 par la sociologue norvégienne Kari Waerness.

39 Afin d'esquisser une approche conceptuelle, Waerness prend comme point de départ les significations que la notion de soin dans le langage quotidien tend à acquérir (dans ce cas, les langues germaniques et particulièrement l'anglais) : s'occuper de et veiller au bien-être d'autrui (taking care of) et se soucier d'elleux (caring for/caring about). Ces deux significations font référence au lien entre (au moins) deux personnes, de même qu'elles évoquent les dimensions de la pratique/de l'action et des affects/émotions présentes dans cette relation.

40 Waerness analyse trois situations sociales dans lesquelles ces relations se manifestent : 1) des liens de réciprocité entre les adultes qui entretiennent des relations personnelles (l'un-e se soucie de l'autre) ; 2) les soins que les femmes procurent à leurs maris, à leurs enfants adultes et à d'autres adultes autonomes de la famille et par conséquent capables de se débrouiller seuls ; 3) les soins que les femmes, en particulier, procurent aux personnes dépendantes (enfants, personnes malades, personnes âgées, personnes en situation de handicap). À partir de ces situations, elle détermine trois types de soin : 1) le soin dans le cadre de relations symétriques ; 2 ) le soin dans le cadre de relations de servilité ; 3) le soin de personnes dépendantes. Or, le troisième type est le seul que l'on peut considérer, selon Waerness, comme un travail de soin qui mérite être questionné dans le domaine des politiques publiques.

41 Dans un effort d'objectivation de la notion de travail de soin, Waerness circonscrit le contenu du concept aux tâches habituellement réalisées par les femmes pour contribuer à la durabilité de la vie des personnes dépendantes - qui ne peuvent pas, ou presque pas, les effectuer - au foyer/famille ou à la sphère publique/étatique. Par ailleurs, elle affirme que les personnes fournissant ces types de soins ont l'envie et l'obligation de réaliser ces activités et font preuve d'empathie, de souci, et d'affection envers la personne soignée.

42 Ces premières approximations de la notion de soin et de travail de soin ont été remises en question par une autre sociologue scandinave, Arnlaug Leira (1994), qui interroge l'idée selon laquelle le soin va nécessairement de pair avec l'amour - en tant que travail d'amour - et que les facultés de soin sont inhérentes aux femmes. Elle ajoute que la notion de travail de soin, ainsi que la conceptualise et la définit Waerness, présente un double contenu normatif : en premier lieu, parce qu'il s'agit d'une obligation de faire quelque chose pour la personne dont on s'occupe, et en second lieu, parce que c'est une activité imprégnée d'un sentiment spécial d'inquiétude ou de dévotion envers celle-ci.

43 Selon Leira, Waerness ne réussit pas à se détacher de la prénotion selon laquelle prodiguer des soins est une expression «naturelle » de l'amour que l'on porte à la personne dont on prend soin, même lorsqu'on essaye d'objectiver ses relations comme du «travail». L'expérience quotidienne démontre que les activités de soin ne s'entremêlent pas nécessairement avec des sentiments d'amour et de dévotion : ainsi, l'existence d'un lien a priori entre l'action et divers affects dans les activités de soin est remise en question. Bien au contraire, quand elle est conceptualisée comme travail, la 
réalisation de soins requiert des aptitudes et des capacités qui ne sont pas naturelles, mais qui s'acquièrent à travers différents processus d'apprentissage.

44 À partir des années 1980, les féministes scandinaves encouragent les débats à propos de la frontière entre la prise en charge publique ou privée du soin, de son caractère rémunéré versus non rémunéré, des conséquences sur l'égalité de genre par la redistribution du soin entre différents acteurs et les institutions (Leira et Saraceno 2006). À partir de ces discussions, la question du soin est entrée dans l'agenda public de ces pays et on a commencé à parler d'État prodigueur de soin (Estado cuidador) (caring state). Des projets missionnés par l'État ainsi que des études concernant cette problématique voient le jour. Un de ces projets menés à bien par une agence gouvernementale suédoise a donné lieu à un rapport intitulé « Un temps pour soigner » (Time to Care), qui a été publié en anglais en 1984. Ce rapport marque sans aucun doute un moment important dans la diffusion internationale de la notion de soin au sein de l'espace européen: on peut lui ajouter les conférences prononcées sur le sujet à Helsinki (Finlande) en 1987 et 1989 (Balbo 2005).

45 Le rapport susmentionné fait le lien entre les préoccupations des féministes scandinaves et les recherches des sociologues italiennes consacrées à l'étude de la vie quotidienne et de la famille (Laura Balbo, Franca Bimbi, Chiara Saraceno, entre autres). Ce document est présenté en Italie, après quoi Laura Balbo en traduit le résumé (Leira et Saraceno 2006) en 1987 et publie un livre en italien, dont le titre inclut à nouveau l'expression anglaise " time to care».

46 Laura Balbo s'était déjà fait connaitre à la fin des années 1970, en ajoutant une nouvelle catégorie à l'analyse du quotidien des femmes sur le marché du travail : la double présence (doppia presenza). Cette catégorie saisit la relation temps-travail avec plus de clarté que la double charge (double burden), définie par la sociologue anglaise Ann Oakley en 1974, ou que la double journée (double shift), un concept d'Arlie Hochschild datant de la fin des années 1980, dans la mesure où elle montre le mode synchronique de répartition des temps, des responsabilités et des activités des femmes qui font un quelconque travail salarié sur le marché, en même temps qu'elles s'occupent des tâches du foyer. On ne parle plus ici de temps linéaires, diachroniques : la double présence renvoie à un temps élargi qui devient diffus, qui inclut des temps parallèles, entrecroisés, superposés et simultanés.

47 Ce que les chercheurs/ses italiennes mettent au cœur même de la question, c'est le temps que demande la réalisation des tâches de soin et la façon dont les femmes l'appréhendent subjectivement (Carrasco, Borderías y Torns 2011). Par ailleurs, elles empruntent le terme utilisé en italien pour faire référence au travail de soin : lavoro di cura, en liant ainsi les tâches de soins à la notion de travail.

48 La perspective italienne nourrit les problématiques théorico-pratiques des chercheurs/ses scandinaves, en cela qu'elle réussit à lier le travail avec le temps/les temps, et qu'elle initie un des axes de recherche les plus féconds dans le domaine du soin : les études sur l'usage du temps dédié à ces activités.

49 En Angleterre, la première étude sur le soin est attribuée aux sociologues Janet Finch et Dulcie Groves, qui publient Un travail d'amour : femmes, travail et soin (À Labour of Love: Women, Work and Caring). Ce texte, publié en 1983 (un an avant celui de Kari Waerness), aborde de façon relationnelle le concept de soin et souligne que l'exécution de ces activités implique un engagement, des obligations, de la confiance, de la loyauté, en soulignant ainsi les composantes émotionnelles présentes des tâches effectuées 
principalement par les femmes et qui ne sont pas assimilables au travail de production de marchandises, principalement masculin (Daly et Lewis 2000; Carrasco, Borderías et Torns 2011).

50 Mary Daly et Jane Lewis (2000) ont inventé la notion de soin social (social care) dans le but d'offrir un outil théorique pour analyser sur un plan plus général les Étatsprovidence. Les auteures soulignent la nature multidimensionnelle de ce concept en insistant sur trois dimensions: le soin comme une forme particulière de travail : la dimension normative du soin qui s'inscrit dans un tissu d'obligations et de responsabilités : les aspects liés au coût économique et émotionnel de ces tâches. Par conséquent, les sociologues anglaises considèrent que le soin renvoie à ces activités et relations qui permettent de répondre aux nécessités physiques et émotionnelles des enfants et des adultes dépendant-es, ainsi que les cadres normatifs, économiques et sociaux au sein desquels on assigne et effectue ces tâches (Daly et Lewis 1000:285; Esquivel, Faur et Jelin $2012: 17$ ). Cette définition fera partie de celles qui se répandront en Amérique latine.

51 En plus de montrer les principales dimensions du concept, les auteures anglaises préviennent des dualités qui le composent, telles que le soin formel versus le soin informel, rémunéré versus non rémunéré, porté aux enfants versus porté aux adultes ou aux personnes âgées. Elles proposent de dépasser ces divisions et d'exploiter le potentiel heuristique du concept pour relier différentes sphères, afin de mieux comprendre le fonctionnement des États-providence.

\section{Traverser l'Atlantique : quelques abordages théorico- empiriques du soin en Amérique latine}

52 Les développements autour du concept de soin dans les pays occidentaux ont été traduits de façon quasi automatique et acritique dans notre région, avec quelques différences quant aux variables de l'analyse empirique, bien qu'on entrevoie ces dernières années quelques élaborations théoriques et enquêtes empiriques qui cherchent à retrouver les particularités du contexte latino-américain et qui tentent de conceptualiser le soin à partir d'un regard propre (à ce sujet, voir Iconos, Revista de Sciences Sociales ${ }^{\circ} 50$, FLASCO - Dossier sur les Nouvelles approches sur l'organisation sociale du soin. Débats latino-américains).

53 Il est important de se rappeler, à ce stade, que la notion de soin/travail de soin émerge dans le contexte de la réalité des pays industrialisés occidentaux, dans la mesure où le schéma dominant de la reproduction s'est configuré autour du modèle de la famille nucléaire hétérosexuelle, avec un seul pourvoyeur et une division sexuelle claire du travail (l'homme pourvoyeur et la femme au foyer). Ce schéma s'est transformé en un modèle de double pourvoyeur avec deux salaires inégaux, conséquence de l'insertion croissante des femmes dans le travail salarié (Ravazi, cité dans Vega et Guttiérrez Rodriguez 2014). Vega et Guttiérrez Rodriguez (2014) soulignent que dans les pays périphériques, l'organisation du maintien de la vie et de la reproduction répond à des configurations diverses, marquées de fortes hétérogénéités régionales, ruralesurbaines, économiques, ethniques, etc.

54 Ces dernières années, dans différents pays d'Amérique latine (Argentine, Uruguay, Brésil, Colombie, Équateur, Bolivie, entre autres), on a pu remarquer une augmentation 
nette d'écrits sur le soin, qui montrent des degrés d'autonomie variables par rapport aux approches européennes: depuis des études qui utilisent simplement les outils conceptuels eurocentrés, jusqu'à des travaux qui tentent de redéfinir le soin dans le cadre de la pensée décoloniale qui questionne les notions de développement et de providence émanant des théories sociales andro- et eurocentrées.

55 Dans cette lancée, nous nous concentrons sur une publication collective sur le soin des enfants (le livre intitulé Les logiques du soin des enfants. Entre la famille, l'État et le marché) qui fait appel à deux sociologues argentines reconnues, Elizabeth Jelin et Eleonor Faur, avec une chercheuse argentine en économie féministe, Valeria Esquivel. D'autre part, nous nous arrêtons sur quelques travaux élaborés par la sociologue uruguayenne Karina Batthyany et son équipe d'enquête.

56 Les auteures argentines se focalisent spécifiquement sur la problématique du soin des personnes, qui s'est imposée comme un champ thématique émergent, ayant pris de l'ampleur dans les dernières décennies dans les pays en développement. Cherchant à retracer l'histoire de la conceptualisation du soin, elles signalent que c'est le débat autour de la séparation entre les processus sociaux de production liés au marché, et les processus de reproduction dans le cadre domestique qui en constitue le point de départ. Cette différenciation caractéristique du capitalisme rend invisible, du point de vue économique, le travail domestique et le subordonne au travail régi par les échanges marchands, de même qu'elle renforce la structure patriarcale de la société à travers le modèle du foyer nucléaire où le travailleur homme apporte les ressources financières pour le maintien de la famille, alors que la femme au foyer-mère transforme les entrées d'argent en biens et services qui rendent possible la reproduction sociale.

L'une des notions centrales exposées dans la publication mentionnée ci-dessus est l'organisation sociale $d u$ soin, que l'on peut associer à la classification des Étatsprovidence élaborée par le sociologue danois Gosta Esping-Andersen. L'intérêt de ce concept est de montrer la manière dont l'État, le marché et la famille sont imbriqués dans la logique du soin, en même temps qu'il permet de signaler que toute politique sociale suppose une certaine forme d'organisation sociale du soin. À ce propos, les auteures font référence au concept de « diamant du soin » (diamante de cuidado), forgé par Shahra Razavi, spécialiste d'origine iranienne en études de genre, qui souligne la présence de quatre acteurs dans la prise en charge du soin, à savoir l'État, la famille, le marché et la communauté, et met par ailleurs en évidence les relations entre eux. Or, les auteures préfèrent l'utilisation de la notion d'organisation sociale du soin, car elles considèrent qu'elle est plus adaptée aux réalités latino-américaines où les politiques de soin sont segmentées et de qualité inégale, donnant lieu à une configuration où existent plusieurs diamants du soin. Dans ce contexte, la démarchandisation et la "défamilisation » du soin sont présentées comme des moyens de résoudre les inégalités de genre et de classe en matière de soin.

De son côté, Valeria Esquivel (2011, 2008), l'une des principales représentantes de l'économie féministe qui travaille sur la question du soin dans notre région, propose de parler d'économie du soin en soutenant l'idée qu'à travers cette notion, nous pouvons dépasser l'abstraction des concepts tels que le travail domestique, le travail reproductif et le travail de soin. Ce qu'elle appelle l'économie du soin permet d'intégrer deux sphères : celle de l'économie, du marché et de la production, ainsi que celle du soin associé à l'intime et au quotidien. Par ailleurs, l'économie du soin met l'accent aussi 
bien sur le bien-être de celleux qui bénéficient du soin que sur les couts associés à celleux qui le prodiguent.

59 Ce concept permet d'observer comment la prise en charge des personnes dépendantes est assurée à la fois par les ménages, l'État et le marché, et de mettre en évidence deux idées : premièrement, que le soin dans nos sociétés n'est pas dispensé exclusivement à domicile et, deuxièmement, qu'il existe des inégalités d'accès et de prise en charge du soin liées à la classe sociale et au genre.

60 Mais l'intérêt principal de l'économie du soin est, selon l'auteure, qu'elle contribue à définir le soin comme une question de politique publique, en le retirant de la sphère privée et en niant sa nature propre aux femmes et aux foyers. On pourrait dire que l'économie du soin propose une vision intégrale, dans laquelle il s'agit non seulement de mesurer, d'estimer et d'intégrer le soin dans les analyses économiques, mais aussi de remettre en question le fonctionnement même du système économique.

61 Karina Batthyány (2007 a, 2007b, 2004, 2001), chercheuse uruguayenne et pionnière dans l'approche de ce sujet, élargit le concept du soin en l'associant à toutes les actions qui visent à assister physiquement, psychologiquement et émotionnellement les êtres humains, selon leur appartenance aux différents groupes d'âge. Elle considère également que l'attribution exclusive de ces tâches aux femmes nuit au développement de leur citoyenneté sociale. L'assignation des tâches reproductives a varié au cours de l'histoire, selon les différents modes de production, mais le rôle principal a toujours été donné aux femmes, reléguées à la sphère domestique et confrontées à de grandes difficultés pour participer à la sphère publique. L'auteure développe différents indicateurs pour analyser cette inégalité. En premier lieu, elle propose l'analyse des sphères sociales constituant les domaines d'activité des femmes et des hommes. Le deuxième indicateur est le degré d'institutionnalisation de l'égalité, de l'inégalité ou de la complémentarité des genres dans la société. Le troisième indicateur met en évidence le niveau de participation du secteur public aux tâches de soin, c'est-à-dire la sphère sociale à laquelle une société attribue le soin (publique/privée). Le dernier indicateur mesure l'importance relative des couples monogames par rapport aux autres modes de vie.

62 En Uruguay, selon l'analyse réalisée par l'auteure, les politiques publiques menées par les États-providence de la région, bien qu'elles aient abordé la question du soin dans le cadre public, n'ont pas permis d'adopter une position critique contre la conception féminisée du travail reproductif.

63 Outre ces approches théoriques, les auteures citées précédemment ont élaboré une série d'études quantitatives et qualitatives qui tentent de tenir compte de la multiplicité des configurations du soin et de leurs stratégies respectives.

64 Les études quantitatives sont fondées sur des données issues d'enquêtes sur l'usage du temps. À cet égard, Valeria Esquivel a coordonné l'élaboration et la mise en œuvre d'une étude inédite dans notre pays, en Argentine : «L'enquête sur l'usage du temps dans la ville de Buenos Aires ", réalisée en 2005.

65 Dans la publication collective dont nous parlions plus haut (Esquivel, Faur et Jelin 2012), un chapitre est dédié à l'analyse des principaux résultats de cette enquête, qui place la focale sur la relation entre les temps consacrés par les mères, les pères et autres proches au soin des enfants, et le temps consacré au travail reproductif. Cette analyse vise à rendre compte des tensions à l'intérieur des foyers entre le temps dédié 
au travail rémunéré et au travail de soin, en essayant de comprendre les manières dont les foyers résolvent ou non ces tensions. À travers les données, il s'agit de mettre en lumière les facteurs qui déterminent l'usage différencié des temps par les composantes du foyer, ainsi que les rythmes de travail quotidien des pères et des mères. Cette analyse montre la tension entre le travail rémunéré et celui du soin, en mettant en évidence les inégalités de genre et d'accès au marché du travail et aux institutions pourvoyeuses de soin.

66 Dans le contexte uruguayen, Karina Batthyany, avec son groupe de recherche, réalise une analyse détaillée des enquêtes sur l'usage du temps, effectuées durant les années 2001, 2007 et 2013 dans son pays. Ces enquêtes examinent, dans une période de référence spécifique, les tâches que les personnes réalisent dans les sphères publiques et privées : il s'agit en effet d'un instrument important pour mesurer le bien-être de la population, ainsi que les inégalités sociales et de genre. Les conclusions de cette analyse signalent, à grands traits, que la charge féminine globale de travail est plus grande que celle des hommes, en ce sens où ceux-ci participent moins aux tâches domestiques. De cette manière, le temps du travail rémunéré est toujours inférieur chez les femmes que chez les hommes. Si on croise ces résultats avec la classe, les foyers populaires présentent des fossés plus importants d'inégalité de genre. L'auteure élargit l'application des indicateurs qui mesurent l'inégalité dans le contexte spécifique de son pays.

67 Les enquêtes sur l'usage du temps réussissent à mettre en évidence les inégalités dans la participation et le temps dédié au soin par les différentes composantes du foyer. Cependant, nous considérons qu'elles ont pour principal manque de ne pas réussir à mettre en lumière la manière dont se prennent les décisions à l'intérieur des ménages pour mettre en place diverses stratégies d'organisation du soin, depuis la perspective des sujets engagés. Pour rendre compte de cela, il a été nécessaire de développer des études de nature qualitative.

68 Il est intéressant de mentionner le travail réalisé par Eleonor Faur, intitulé « Le soin des enfants depuis la perspective des femmes-mères» («El cuidado infantil desde las perspectivas de las mujeres-madres»), et incorporé à une publication collective sur le soin des enfants (Esquivel, Faur et Jelin 2012). Dans cette étude, l'auteure aborde le thème de l'organisation sociale du soin et essaye de comprendre la manière dont se prennent les décisions à l'intérieur du foyer pour développer les différentes stratégies familiales du soin. À travers l'analyse de données qualitatives provenant d'entretiens réalisés dans deux quartiers populaires de Buenos Aires, l'auteure a pu identifier quatre situations relatives au soin des enfants jusqu'à 5 ans : les mères qui s'occupent du soin à temps complet, le soin pris en charge par d'autres membres de la famille, l'accès à des services publics de soin et la marchandisation du soin, que cela soit à travers des crèches privées ou des services de garde à domicile.

69 À travers ce travail, Faur met en évidence que ce sont les femmes qui sont soumises à la tâche difficile de concilier le travail productif et reproductif, c'est-à-dire le temps où elles s'occupent de la famille et leur participation au marché du travail. Pour cela, l'auteure assigne un rôle fondamental à l'État qui, à travers ses politiques, peut intervenir dans l'organisation sociale du soin, accentuant, ou, au contraire, diminuant les inégalités de genre et de classe. Plus les familles se voient attribuer les responsabilités du soin de l'enfant, plus on charge les femmes. Au contraire, à mesure qu'augmente l'offre de services publics de soin de la part de l'État, on facilite la 
"défamilisation » et la "démarchandisation » de ceux-ci, en tendant à réduire les inégalités de genre et de classe.

\section{Conclusion}

70 Dans cette présentation, nous avons signalé l'importance de reconnaître que tout concept est une construction socio-historique et que ses usages, tant scientifiques que quotidiens, requièrent un effort constant de dénaturalisation et d'ancrage dans un processus socio-historique spécifique.

71 En ce sens, nous avons effectué un bref passage par quelques approches théoriques qui renvoient à la notion de travail et questionné la définition restreinte de celle-ci, qui se focalise seulement sur l'activité économique rémunérée réalisée dans le domaine marchand. Cette conceptualisation invisibilise et dévalorise d'autres types de travails, parmi lesquels le travail domestique et plus généralement, le travail reproductif.

72 Dans les dernières décennies surgit le concept de soin /travail de soin, qui a acquis une acception assez ample dans les études de genre et sur le travail des femmes. Dans notre travail, nous réalisons une exploration de la genèse de cette notion, en rendant compte de ses premières origines et acceptions, comme elles ont été développées dans la sociologie et la science politique féministe scandinave, dans la sociologie de la vie quotidienne italienne et dans la sociologie anglaise.

73 Pour finir, nous nous sommes concentré-es sur les manières dont la notion de soin a circulé en Amérique latine (en particulier en Argentine et en Uruguay), en mettant l'accent sur quelques usages conceptuels et applications empiriques qui se sont développées dans la sociologie et l'économie féministe de la région.

74 Les travaux que nous sommes actuellement en train de mener à bien dans cet axe de recherche ont vocation à se poursuivre pour approfondir le débat en le nourrissant d'études théoriques qui explorent et clarifient les différentes acceptions qu'ont revêtu les notions de travail reproductif et de soin depuis les différentes perspectives utilisées en Amérique latine, ainsi que de recherches empiriques qui contribuent à recueillir de nouvelles données, pour approfondir la compréhension de cette problématique dans la région.

\section{BIBLIOGRAPHIE}

ALESTALO, Matti et al. 2009. " The Nordic Model: Conditions, Origins, Outcomes, Lessons ». Hertie School of Governance, Working Papers 41.

BALBO, Laura .2005. « Making a European Quilt. "Doing Gender" in The European Social Sciences ». The Ursula Hirschmann Anual Lecture on Gender and Europe. Florence : European University Institute. 
BALBO, Laura. 1994. « La doble presencia » in Las mujeres y el trabajo, BORDERÍAS, Cristina et al. (éds.). Barcelona : ICARIA, 503-514.

BATTHYÁNY, Karina. 2007a. « Articulación entre vida laboral y vida familiar. Las prácticas de cuidado infantil de trabajadoras asalariadas de Montevideo » in Género, familias y trabajo: rupturas y continuidades: desafíos para la investigación política, GUTIERRÉZ, María Alicia (éd.). Buenos Aires : CLACSO, 137-168.

BATTHYÁNI, Karina. 2007b. Género y cuidados familiares. ¿Quién se hace cargo del cuidado y la atención de los adultos mayores en Montevideo? Informe de investigación. Montevideo : Facultad de Ciencias Sociales, Universidad de la República, Montevideo.

BATTHYÁNY, Karina. 2004. Cuidado infantil y trabajo: ¿un desafío exclusivamente femenino? Una mirada desde el género y la ciudadanía social. Montevideo : CINTERFOR.

BATTHYÁNY, Karina. 2001. «El trabajo de cuidados y las responsabilidades familiares en Uruguay: proyección de demandas » in Trabajo, género y ciudadanía en los países del Cono Sur, AGUIRRE, Rosario \& BATTHYANY, Karina (coord.). Montevideo : Cinterfor, 223-242.

BOURDIEU, Pierre et al. 1983 [1968]. Le métier de sociologue. Préalables épistemologiques. La Haye : Mouton Editeur.

BENERÍA, Lourdes. 1999. « El debate inconcluso sobre el trabajo no remunerado » Revista Internacional del Trabajo 118(3) : 321-346.

BENERÍA, Lourdes. 1979. « Reproduction, production and the sexual division of labour » Cambridge Journal of Economics 3(3) : 203-225.

CARRASCO, Cristina, BORDERÍAS, Cristina \& TORNS, Teresa (éds.). 2011. El trabajo de cuidados. Historia, teoría y políticas. Madrid : Catarata.

CARRASQUER, Pilar, TORNS, Teresa et al. 1998. « El trabajo reproductivo » Papers, Revista de Sociologia 55 : 95-114.

DALY, Mary \& LEWIS, Jane. 2000. « The concept of social care and the analysis of contemporary welfare states » British Journal of Sociology, 51(2) : 281-298.

DE BARBIERI, Teresita. 1984. Mujeres y vida cotidiana. México : FCE.

DE BARBIERI, Teresita. 1978. « Notas para el estudio del trabajo de las mujeres: el problema del trabajo doméstico » Demografía y Economía 12(1) : 129-137.

DE LA GARZA, Enrique. 2008. Hacia un concepto ampliado de trabajo. México : UAM.

DE LA GARZA, Enrique. 2005. «Del Concepto Ampliado de Trabajo al de Sujeto Laboral Ampliado » in Sindicatos y nuevos movimientos sociales en América Latina, DE LA GARZA, Enrique. Buenos Aires : CLASCO.

DE LA GARZA, Enrique. 2001. « Problemas clásicos y actuales de la crisis del trabajo » in El trabajo del futuro. El futuro del trabajo, NEFFA, Julio \& DE LA GARZA, Enrique (éds.) Buenos Aires : CLACSO.

ESQUIVEL, Valeria, FAUR, Eleonor \& JELIN, Elizabeth (éds). 2012. Las lógicas del cuidado infantil. Entre las familias, el Estado y el mercado. Buenos Aires : IDES.

ESQUIVEL, Valeria. 2011. La economía del cuidado en América Latina: poniendo a los cuidados en el centro de la agenda. Collection de cahiers : « Atando cabos, desatando nudos ». s.l. : PNUD.

ESQUIVEL, Valeria. 2008. The Political and Social Economy of Care: Argentina Research Report 2. s.l. : UNRISD. 
FAUR, Eleonor. 2012. « El cuidado infantil desde las perspectivas de las mujeres-madres. Un estudio de dos barrios populares del Área Metropolitana de Buenos Aires » in Las lógicas del cuidado infantil. Entre las familias, el Estado y el mercado, ESQUIVEL, Valeria, FAUR, Eleonor \& JELIN, Elizabeth (éds). Buenos Aires : IDES.

FAUR, Eleonor. 2006. « Género y conciliación familia-trabajo: legislación laboral y subjetividades masculinas en América Latina » in Cohesión social, políticas conciliatorias y presupuesto público. Una mirada desde el género, MORA, Luis et al. (éds.).. México DF : UNFPA-GTZ.

FEDERICI, Silvia. 2013. Revolución en punto cero. Trabajo doméstico, reproducción y luchas feministas. Madrid : Traficantes de Sueños

FEDERICI, Silvia. 1975. Wages against Housework. Bristol : Falling Wall Press.

FOLBRE, Nancy \& NELSON, Julie A. 2000. « For Love or Money - or Both » Journal of Economic Perspectives 14(4) : 123-140.

FOLBRE, Nancy. 1986. « Hearts and Spades: Paradigms of Household Economics » World Development 14(2) : 245-255.

GUTIÉRREZ, María Alicia (éd.). 2007. Género, familias y trabajo: rupturas y continuidades: desafíos para la investigación política. Buenos Aires : CLACSO.

HIMMELWEIT, Susan. 2005. « El descubrimiento del trabajo no pagado: las consecuencias sociales de la expansion del trabajo » in Debate sobre el trabajo doméstico. Antología, RODRIGUEZ, Dinah \& COOPER, Jennifer (éds.). México : UNAM.

HOCHSCHILD, Arlie. 1990. The second shift. Nueva York : Avon Books.

JELIN, Elizabeth. 2008. « Gender and the family in public policy: a comparative view of Argentina and Sweden » in Global perspectives on gender equality. Reversing the gape, KABEER, Naila \& STARK, Agneta (éds). New York and London : Routledge/EGDI.

KABEER, Naila. 1998. Realidades trastocadas. Las jerarquías de género en el pensamiento del desarrollo. Madrid : Paidós.

LARGUÍA, Isabel \& DUMOULIN, John. 1976. Hacia una ciencia de la liberación de la mujer. Barcelona : Anagrama.

LEIRA, Arnlaug \& SARACENO, Chiara. 2006. « Care: actors, relationships, contexts » Sosiologi I Dag 36(3) : 7-34.

LEIRA, Arnlaug. 1994. « Concepts of Caring: Loving, Thinking and Doing » Social Service Review 68(2) : 185-201.

MIES, Maria. 1981. «The Social Origins of Sexual Division of Labour » ISS Occasional Papers. La Hague : Institute of Social Studies, Netherlands.

OLIVEIRA, Orlandina \& ARIZA, Marina. 1999. « Trabajo femenino en América Latina: una revisión de las principales perspectivas analíticas » in Repositorio digital REDALYC, http:// repositoriodigital.academica.mx/jspui/handle/987654321/352917

OLIVEIRA, Orlandina \& SALLES, Vania. 1987. « Reflexiones teóricas para el estudio de la reproducción de la fuerza de trabajo ». Intervention à la 2è réunion SOMEDE, México.

RODRIGUEZ, Dinah \& COOPER, Jennifer (éds.). 2005. Debate sobre el trabajo doméstico. Antología. México : UNAM.

SECCOMBE, Wally. 1974. «El trabajo del ama de casa en el capitalismo » New Left Review. I/83 : s.p. 
TORNS MARTÍN, Teresa. 2008. «El trabajo y el cuidado: cuestiones teórico-metodológicas desde la perspectiva de género » EMPIRIA Revista de Metodología de Ciencias Sociales 15 : 53-73.

TORNS MARTÍN, Teresa. 2005. « De la imposible conciliación a los permanentes malos arreglos » Cuadernos de Relaciones Laborales 23(1) : 15-33.

TORNS MARTÍN, Teresa. 2001. « La doble presencia: ¿una propuesta para lograr la conciliación? » Intervention aux Journées Doble jornada-Doble presencia, Pamplona, 17 octobre 2001.

VEGA, Cristina \& GUTIÉRREZ RODRÍGUEZ, Encarnación. 2014. « Nuevas aproximaciones a la organización social del cuidado. Debates latinoamericanos. Presentación del Dossier » Iconos, Revista de Ciencias Sociales 50 : 9-26.

WAINERMAN, Catalina (éd.). 2002. Familia, trabajo y género. Un mundo de nuevas relaciones. Buenos Aires : FCE/UNICEF.

WAERNESS, Kari. 1996. "The Rationality of Caring » in Caregiving. Readings in Knowledge, Practice, Ethics and Politics, GORDON, Suzanne et al. Philadelphia : University of Pennsylvania Press :

231-255

\section{NOTES}

1. NdT : cuidado/cuidados: Le concept de soin, au sens féministe du terme, vient des études anglophones, et notamment des travaux de Carol Gilligan (voir In a Different Voice: Psychological Theory and Women's Development, Cambridge MA : Harvard University Press, 1982). L'auteure y oppose la perspective de la justice comme modèle du développement moral des personnes assignées hommes, et la perspective du care comme modèle du développement moral des personnes assignées femmes, encouragées à s'occuper des autres et à entretenir les relations au sein de foyer et dans les différentes sphères sociales. Dans les textes francophones, le terme reste souvent non traduit, car le soin en français a une acception plus réduite, et se limite principalement à la sphère médicale. Cependant, malgré les limites du terme soin, nous choisissons de le conserver car il nous parait important, tout en rappelant le contenu du concept de care, de proposer des traductions intégralement en français. Dans le présent article, les auteur-es emploient à la fois les termes cuidado et cuidados. Il semble que les occurrences de cuidados ont une portée plus abstraite et renvoient au concept anglais de care, tandis que les occurrences de cuidado font référence à l'activité plus restreinte et spécifique du soin des personnes. Ainsi, nous avons choisi de traduire, paradoxalement, cuidados par le soin (comprendre, le care), et cuidado par les soins.

\section{RÉSUMÉS}

La notion de travail, comme d'autres concepts, revêt des significations différentes en fonction des perspectives théoriques depuis lesquelles on la définit, de telle sorte que ce n'est pas un concept anhistorique, mais une construction sociale modelée par les relations de pouvoir et de domination à l'œuvre dans un ordre social déterminé. Ainsi, dans le mode de production capitaliste, la notion de travail s'est limitée aux activités qui produisent des biens et des services 
dotés d'une valeur d'échange sur le marché, c'est-à-dire les activités qui contribuent directement à l'accumulation du capital. Les formes de travail qui s'éloignent de la définition dominante furent marginalisées dans la pensée conceptuelle orthodoxe des sciences sociales comme dans le champ empirique, avec pour conséquence l'impossibilité de quantifier ce phénomène. Il a fallu et il faut encore des débats importants pour que des modalités de travail comme celui de la subsistance, du domestique, du bénévolat, de l'autoconsommation, soient considérées comme travail pour commencer à mieux les mesurer empiriquement et mieux les valoriser économiquement. La définition réduite du travail a conduit à la consolidation $\mathrm{du}$ travail rémunéré comme objet d'étude des différentes disciplines des sciences sociales, et a limité ce qu'on appelle le monde du travail aux phénomènes qui entrent dans le champ de l'activité marchande et rémunérée. L'intégration d'une définition plus large qui permettrait de réinsérer les autres formes de travail qui ne se développent pas dans le domaine marchand et la problématisation du travail reproductif comme objet d'étude des sciences sociales se sont mises en place lentement. En effet, l'invisibilisation par le capitalisme d'autres formes de travail qui ne seraient pas le travail rémunéré/salarié a aussi affecté les conditions de possibilité de son appréhension théorico-conceptuelle. Dans les années 1980, une nouvelle notion jusqu'alors étrangère à la théorie sociale a commencé à prendre forme dans les sciences sociales européennes: le soin, ou le travail de soin. Au début, cette notion a trouvé sa place dans les réflexions des sciences sociales et politiques sur les Etats-Providence nord-européens. Elle est devenue vers la fin du XXe siècle un lieu obligé pour les économistes, les féministes, les étudiants et étudiantes en études de genre, qui voulaient dire quelque chose de «nouveau » sur le travail reproductif «traditionnel » et « quotidien » des femmes. Ensuite, la notion a traversé l'océan et commencé à se diffuser en Amérique latine, souvent sous un vernis anglophone : care work. Dans cette communication, nous voulons d'une part proposer un examen critique des différentes définitions du travail de soin qui ont été élaborées dans les pays du centre. D'autre part, il nous intéresse de regarder la circulation du terme en Amérique latine, en interrogeant ses succès et sa pertinence, et en mettant en garde contre une application presque automatique et dénuée d'esprit critique d'une notion qui trouve ses origines dans les débats concernant les politiques propres aux Etats-Providence occidentaux.

La noción de trabajo, al igual que otros conceptos, adquiere significados diferentes de acuerdo a los enfoques teóricos desde los cuales se lo define, de modo que no es un concepto ahistórico, sino que se trata de una construcción social moldeada por las relaciones de poder y dominación vigentes en una determinada formación social. Así, en el modo de producción capitalista, la noción de trabajo se ha circunscripto a las actividades que producen bienes y servicios con un valor de cambio en el mercado, o sea, aquellas que aportan directamente a la acumulación del capital. Aquellas formas de trabajo que se alejan de la definición dominante, fueron marginadas tanto del acervo conceptual ortodoxo de las ciencias sociales, como de la posibilidad de su medición en el plano empírico. Modalidades de trabajo como el de subsistencia, el doméstico, el reproductivo, el voluntario, el trabajo para el autoconsumo, requirieron y siguen requiriendo de extensos debates para ser considerados como trabajo y para poder avanzar, de modo incipiente, en su medición empírica y valoración económica. La conceptualización restringida del trabajo llevó a que se consolidara el trabajo remunerado como objeto de estudio de las diferentes disciplinas de las ciencias sociales, y circunscribió el llamado mundo del trabajo a los fenómenos que se dan en torno de la actividad laboral mercantil y remunerado. La incorporación de una definición ampliada que permita recuperar las otras formas del trabajo que no se desarrollan en el ámbito mercantil y la problematización del trabajo reproductivo en cuanto objeto de estudio de las ciencias sociales, se fue dando lentamente. En efecto, la invisibilización que se produjo en el capitalismo de otras formas de trabajo que no fuesen el remunerado/asalariado, también afectó las posibilidades de su aprehensión teórico-conceptual. En los años '80, en las ciencias 
sociales europeas comenzó a tomar cuerpo una noción nueva, hasta entonces ajena a la teoría social: los cuidados y los trabajos de cuidados. A principios, se instaló en los enfoques sociológicos y politológicos sobre los estados de bienestar del norte europeo, para transformarse hacia fines del siglo XX en un lugar obligado para economistas, feministas, estudiosas/os de la perspectiva de género, que pretendían decir algo "nuevo" sobre el "tradicional" y "cotidiano" trabajo reproductivo de las mujeres. Luego, cruzó el océano y empezó a ser vehiculizada en América Latina, a menudo con ropaje inglés: care work. En esta ponencia pretendemos, por un lado, examinar críticamente las diferentes conceptualizaciones que se fueron desarrollando con respecto al trabajo de cuidados en los países centrales. Por otro lado, nos interesa rastrear algunos recorridos del concepto en América Latina, problematizando sus alcances y aplicabilidad y advirtiendo un traslado cuasi-automático y acrítico de una noción cuyos orígenes se encuentran en los debates acerca de los regímenes de bienestar propios de los países centrales.

\section{INDEX}

Thèmes : Recherches

Mots-clés : travail, travail domestique, soin, Amérique latine, sociologie

Keywords : work, domestic work, care, Latin America, sociology 